\title{
Synthesis of Highly Crystalline and Monodisperse Cobalt Ferrite Nanocrystals
}

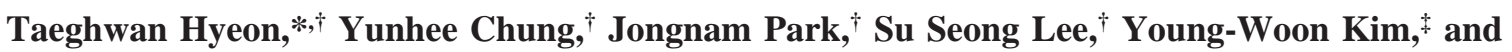 \\ Bae Ho Park ${ }^{\S}$ \\ National Creative Research Center for Oxide Nanocrystalline Materials and School of Chemical Engineering, \\ Seoul National University, Seoul 151-744, Korea, School of Materials Science and Engineering, Seoul National \\ University, Seoul 151-744, Korea, Department of Physics, Konkuk University, Seoul 143-701, Korea
}

Received: April 30, 2002; In Final Form: June 4, 2002

\begin{abstract}
Highly crystalline and monodisperse cobalt ferrite nanocrystals were fabricated by the high-temperature aging of a metal-surfactant complex followed by mild oxidation. Particle sizes were varied from 4 to $9 \mathrm{~nm}$ by changing the experimental conditions. Transmission electron microscopic (TEM) images of the particles showed two- and three-dimensional assembly of the particles, demonstrating the uniformity of the nanocrystals. Electron diffraction, X-ray diffraction, and high-resolution transmission electron microscopic images of the nanocrystals confirmed the highly crystalline nature of the cobalt ferrite structure. The elemental analysis confirmed the stoichiometry of cobalt ferrite, despite some variations in the relative atomic composition of nanocrystals. The nanocrystals were found to have typical behaviors of magnetic nanocrystals and the narrow energy barrier distributions of magnetic anisotropy, implying that the nanocrystals obtained are very uniform.
\end{abstract}

The development of uniform magnetic nanocrystals has been intensively pursued because of their applications in magnetic data storage, ferrofluids, medical imaging, drug targeting, and catalysis. $^{1,2}$ Recently, several metallic magnetic nanocrystals of uniform particle size have been fabricated. ${ }^{3}$ Magnetic oxide nanocrystals having uniform particle size have been synthesized. ${ }^{4,5}$ However, a very difficult size-selection process was required to obtain magnetic nanocrystals having uniform particle size distribution. Recently, we developed a new procedure for producing highly crystalline and monodisperse $\gamma-\mathrm{Fe}_{2} \mathrm{O}_{3}$ nanocrystals without a size-selection process. ${ }^{6}$ These iron oxide nanocrystals were synthesized from the controlled oxidation of uniform iron nanoparticles generated from the thermal decomposition of an iron-surfactant complex. We have extended the synthetic method to fabricate bimetallic oxide nanocrystals and report upon the synthesis of monodisperse and highly crystalline cobalt ferrite nanocrystals.

In the synthetic procedure, uniform iron-cobalt alloy nanoparticles were first generated from the thermal decomposition of a metal-oleate complex and further oxidized to yield cobalt ferrite nanocrystals. The precursor, $\left(\eta^{5}-\mathrm{C}_{5} \mathrm{H}_{5}\right) \mathrm{CoFe}_{2}(\mathrm{CO})_{9}$, was prepared using the previously reported synthetic procedure. ${ }^{7} \mathrm{~A}$ typical synthesis of cobalt ferrite nanocrystals with a particle diameter of $6 \mathrm{~nm}$ is as follows. A total of $0.2 \mathrm{~g}$ of $\left(\eta^{5}-\mathrm{C}_{5} \mathrm{H}_{5}\right)$ $\mathrm{CoFe}_{2}(\mathrm{CO})_{9}(1.23 \mathrm{mmol}$; total metal atoms $)$ was added to a mixture containing $5 \mathrm{~mL}$ of dioctyl ether and $1.04 \mathrm{~g}$ of oleic acid $(3.69 \mathrm{mmol})$ at room temperature under an argon atmosphere. The mixture was heated to reflux $\left(\sim 300^{\circ} \mathrm{C}\right)$ and kept at that temperature for $1 \mathrm{~h}$. The color of the reaction mixture changed to clear blue at $230{ }^{\circ} \mathrm{C}$ and to black at the reflux temperature. The blue color formed at $230{ }^{\circ} \mathrm{C}$ seemed to be due to a metal oleate complex. The resulting black solution was cooled to room temperature, and $0.28 \mathrm{~g}$ of dehydrated $\left(\mathrm{CH}_{3}\right)_{3^{-}}$

* To whom correspondence should be addressed. E-mail: thyeon@ plaza.snu.ac.kr.

School of Chemical Engineering, Seoul National University.

$¥$ School of Materials Science and Engineering, Seoul National University.

$\S$ Department of Physics, Konkuk University.
NO (3.69 mmol) was added. The mixture was then heated to $130{ }^{\circ} \mathrm{C}$ under an argon atmosphere and maintained at this temperature for $2 \mathrm{~h}$, whereupon it formed a dark brown solution. The reaction temperature was slowly increased to reflux, and the reflux was maintained for $1 \mathrm{~h}$; the color of the solution gradually turned from dark brown to black. The solution was then cooled to room temperature, and excess ethanol was added to yield a black precipitate, which was separated by centrifuging. The resulting black powder can be easily redispersed in hydrocarbon solvents, such as hexane, octane, and toluene. Monodisperse nanocrystals were obtained directly without a size-selection process.

The nanocrystals obtained were characterized by electron microscopy, X-ray diffraction, and elemental analysis. The TEM image of the black precipitate obtained from the thermal decomposition of the metal oleate complex showed monodisperse nanocrystals passivated with an oxide layer. The electron diffraction pattern showed a diffuse ring structure, demonstrating the poorly crystalline nature of the nanoparticles. The XRD pattern of the material after heat treatment at $500{ }^{\circ} \mathrm{C}$ under an argon atmosphere showed a bcc structure of an iron-cobalt solid solution. As-synthesized metallic nanoparticles were then transformed into cobalt ferrite nanocrystals by oxidizing them with the mild oxidant trimethylamine $N$-oxide $\left(\left(\mathrm{CH}_{3}\right)_{3} \mathrm{NO}\right)$. The TEM image (Figure 1) showed that the particles were wellseparated and very uniform with diameter of $6 \mathrm{~nm}$. The electron diffraction pattern matched well with standard cobalt ferrite. The uniformity of the nanocrystals was further demonstrated by the formation of a three-dimensional close-packed superlattice assembly (Supporting Information).

To synthesize larger nanocrystals, lauric acid was used in combination with oleic acid as stabilizing surfactant. A total of $0.2 \mathrm{~g}$ of $\left(\eta^{5}-\mathrm{C}_{5} \mathrm{H}_{5}\right) \mathrm{CoFe}_{2}(\mathrm{CO})_{9}(1.23 \mathrm{mmol}$; total metal atoms $)$ was added to a mixture containing $5 \mathrm{~mL}$ of dioctyl ether, 0.35 $\mathrm{g}$ of oleic acid $(1.23 \mathrm{mmol})$ and $0.49 \mathrm{~g}$ of lauric acid $(2.46$ mmol) at room temperature under an argon atmosphere. The rest of the synthetic procedure is very similar to the one applied 


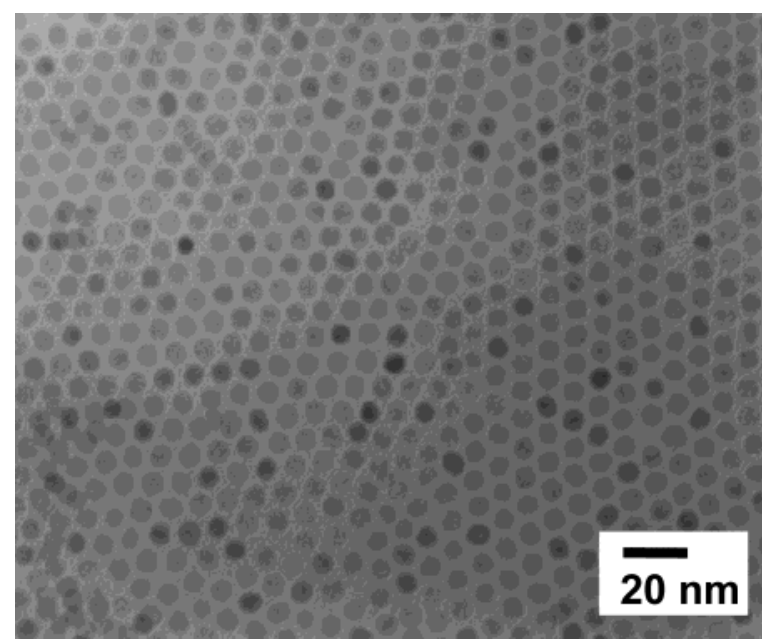

Figure 1. TEM image of a two-dimensional hexagonal assembly of 6 $\mathrm{nm}$ cobalt ferrite nanocrystals. TEM image was obtained on a JEOL EM-2000 EX II microscope.

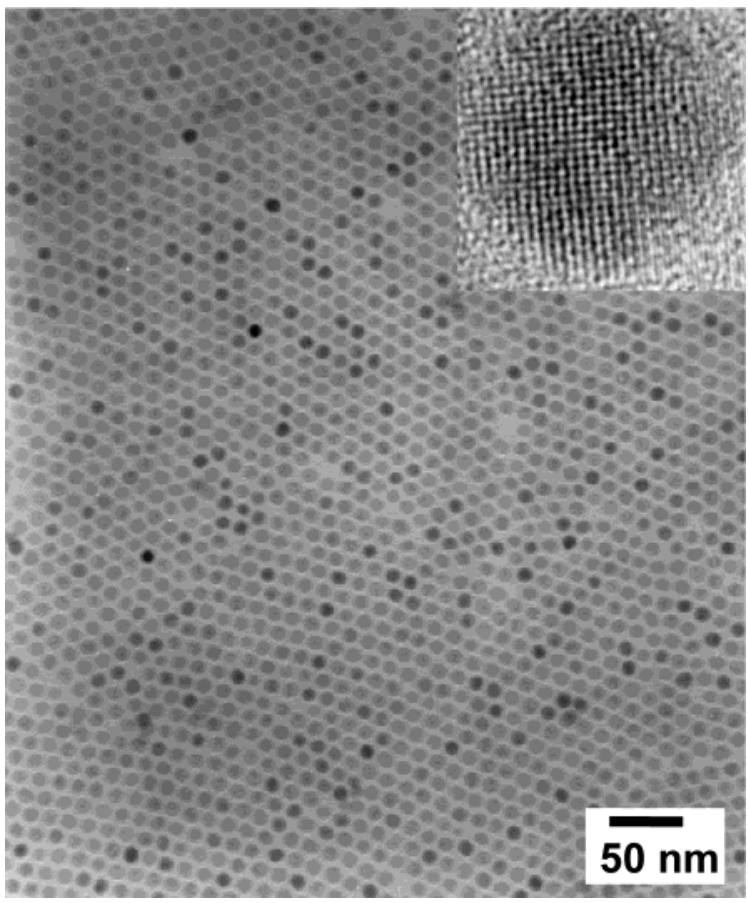

Figure 2. TEM image of a two-dimensional hexagonally close-packed assembly and high-resolution TEM image (inset) of $9 \mathrm{~nm}$ cobalt ferrite nanocrystals. Low-resolution TEM images were obtained on a JEOL EM-2000 EX II microscope, and HRTEM images were taken on a JEOL JEM-3000F microscope.

for the synthesis of $6 \mathrm{~nm}$ cobalt ferrite nanocrystals. Monodisperse nanocrystals were obtained without a further size-selection process. The TEM image (Figure 2) showed that monodisperse nanocrystals with a diameter of $9 \mathrm{~nm}$ were arranged in a twodimensional hexagonal close-packed array, demonstrating the uniformity of the particle size. The high-resolution transmission electron micrograph (HRTEM), shown in the inset of Figure 2 and Supporting Information, demonstrated the highly crystalline nature of the single nanocrystal. The X-ray powder diffraction pattern of the material also proved its highly crystalline cubic spinel structure (Figure 3). Nanoscale elemental analysis of ten 9-nm-sized cobalt ferrite nanocrystals by energy-dispersive X-ray spectroscopy (EDS) showed that the average molar atomic ratio of Fe to Co was 2.3 with a standard deviation of $20 \%$. The EDS data was very well matched by the bulk elemental



Figure 3. X-ray diffraction pattern of $6 \mathrm{~nm}$ cobalt ferrite nanocrystals obtained with a Rigaku D/Max-3C diffractometer equipped with a rotating anode and a $\mathrm{Cu} \mathrm{K} \alpha$ radiation source $(\lambda=0.154056 \mathrm{~nm})$.

analysis results obtained by inductively coupled plasma atomic emission spectrometry (ICP-AES). The EDS result showed that the ratio of iron and cobalt in the precursor was almost unchanged in the final nanocrystals, despite some variations in the relative atomic composition of nanocrystals.

When hexadecylamine and oleic acid were used as stabilizing agents, faceted nanocrystals were obtained. Nanocrystal sizes could be controlled by changing the molar ratio of stabilizing agent to precursor. When the starting reaction mixtures containing the stabilizing agents and the precursor at the molar ratios of $1: 1$ and 1:5 were applied in the synthesis, cobalt ferrite nanocrystals with particle sizes of 4 and $8 \mathrm{~nm}$ were obtained, respectively (Supporting Information). To synthesize $4 \mathrm{~nm}$ nanocrystals, $0.2 \mathrm{~g}$ of $\left(\eta^{5}-\mathrm{C}_{5} \mathrm{H}_{5}\right) \mathrm{CoFe}_{2}(\mathrm{CO})_{9}(1.23 \mathrm{mmol}$; total metal atoms) was used with $0.17 \mathrm{~g}$ of oleic acid $(0.615 \mathrm{mmol})$ and $0.15 \mathrm{~g}$ of hexadecylamine $(0.615 \mathrm{mmol})$. The mixture was refluxed in dioctyl ether and oxidized. In the synthesis, monodisperse nanocrystals could be obtained after a sizeselection process, which employed the addition of ethanol into hexane solution containing a mixture of nanoparticles, followed by separation through centrifugation.

We studied the magnetic properties of the 6 and $9 \mathrm{~nm}$ cobalt ferrite nanocrystals with a superconducting quantum interference device (SQUID) magnetometer. Zero-field-cooled (ZFC) and field-cooled (FC) magnetization data were measured in an applied field of $100 \mathrm{Oe}$ at temperatures between 5 and $300 \mathrm{~K}$, as shown in Figure 4. They exhibited typical behaviors of magnetic nanoparticles. (1) ZFC magnetization $\left(M_{\mathrm{ZFC}}\right)$ increased as the temperature increased from $5 \mathrm{~K}$ and reached a maximum at the blocking temperature $\left(T_{\mathrm{B}}\right)$ and then decreased; (2) FC magnetization $\left(M_{\mathrm{FC}}\right)$ steadily decreased as temperature increased from $5 \mathrm{~K}$; (3) $M_{\mathrm{ZFC}}$ and $M_{\mathrm{FC}}$ overlapped at temperatures over the corresponding $T_{\mathrm{B}}{ }^{3 \mathrm{a}, 8-10}$ The difference between $M_{\mathrm{ZFC}}$ and $M_{\mathrm{FC}}$ below $T_{\mathrm{B}}$ mainly resulted from the existence of energy barriers of magnetic anisotropy. The total magnetic anisotropy constant $(K)$ of magnetic nanocrystals can be determined using the equation $K=25 k_{\mathrm{b}} T_{\mathrm{B}} /\langle V\rangle$, where $k_{\mathrm{b}}$ is the Boltzman constant and $\langle V\rangle$ is the median volume of a single nanocrystal. ${ }^{3 a, 9}$ The calculated $K$ values of our 6 and $9 \mathrm{~nm}$ cobalt ferrite nanocrystals were $5.5 \times 10^{6}$ and $2.0 \times 10^{6} \mathrm{ergs} / \mathrm{cm}^{3}$, respectively. These values were comparable to the $K$ values of $\mathrm{CoFe}_{2} \mathrm{O}_{4}$ bulk materials $\left((1.8-3.0) \times 10^{6} \mathrm{ergs} / \mathrm{cm}^{3}\right){ }^{11}$

Using the methods of Zhang et al., ${ }^{7}$ we were able to obtain the distribution function of the magnetic anisotropy energy 

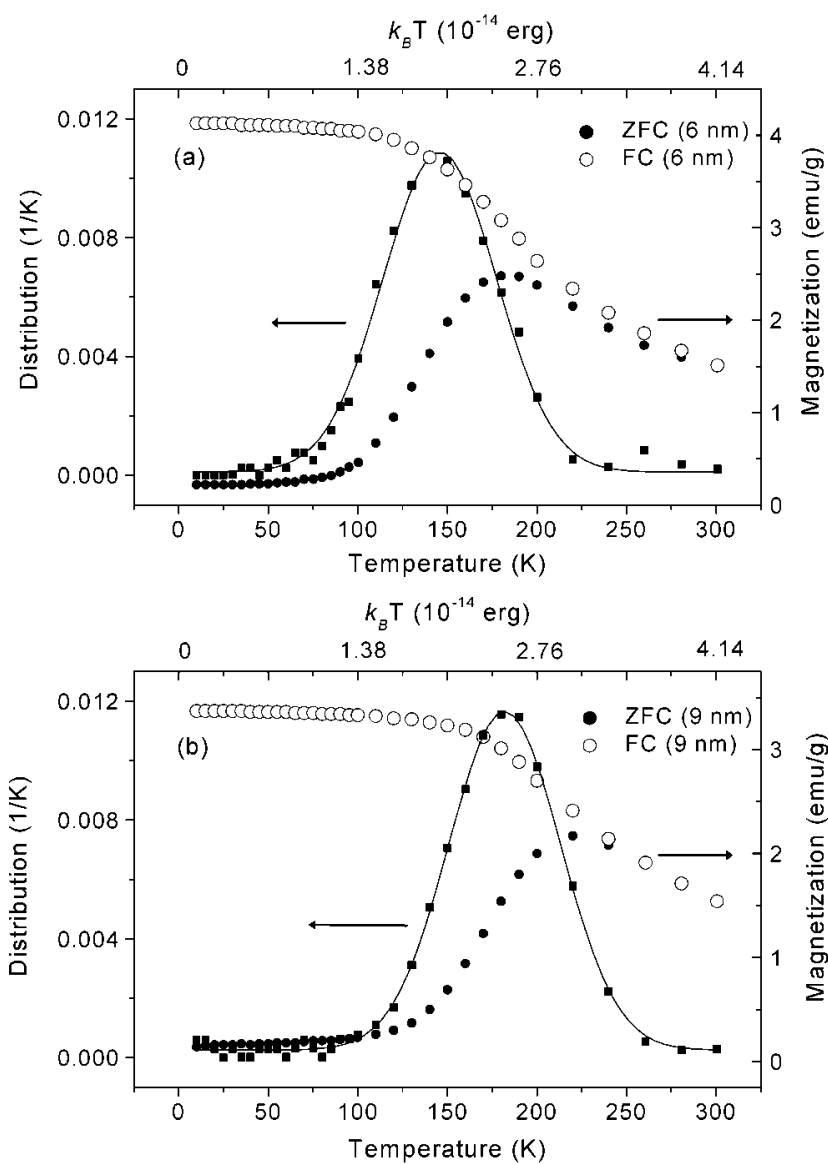

Figure 4. Zero-field-cooled $(\bullet)$ and field-cooled $(O)$ magnetization data measured in an applied field of $100 \mathrm{Oe}$, and energy barrier distributions of magnetic anisotropy (ם) of (a) $6 \mathrm{~nm}$ and (b) $9 \mathrm{~nm}$ cobalt ferrite nanocrystals. The energy barrier distributions of magnetic anisotropy are fitted well using Gaussian functions (solid lines)

barriers, $f(T) . M_{\mathrm{FC}}$ consists of the contribution from all nanoparticles, while $M_{\mathrm{ZFC}}$ reflects only the contribution of nanocrystals of which the energy barriers are overcome by thermal energy at the measuring temperature. Therefore, $M_{\mathrm{ZFC}}, M_{\mathrm{FC}}$, and $f(T)$ satisfy the equation

$$
M_{\mathrm{ZFC}}=M_{\mathrm{FC}} \int_{5}^{T} f\left(T^{\prime}\right) \mathrm{d} T^{\prime}
$$

or equivalently

$$
f(T)=\frac{\mathrm{d}}{\mathrm{d} T}\left(\frac{M_{\mathrm{ZFC}}}{M_{\mathrm{FC}}}\right) .
$$

The calculated anisotropy energy distributions of the 6 and $9 \mathrm{~nm}$ cobalt ferrite nanocrystals are shown in Figure 4 and were fitted using Gaussian functions with center and width values of 145.8 and 63.1 and 182.0 and $62.6 \mathrm{~K}$, respectively. In general, the anisotropy energy distribution depends on the volume and shape distributions of the nanocrystals. Therefore, the narrow anisotropy energy distributions of these nanocrystals imply that the cobalt ferrite nanocrystals produced in this study were more uniform than those reported by others..$^{8,12}$

In conclusion, highly crystalline and monodisperse cobalt ferrite nanocrystals were synthesized by the high-temperature aging of a metal-surfactant complex, followed by mild oxidation. Particle sizes were varied from 4 to $9 \mathrm{~nm}$ by changing the experimental conditions. The synthetic procedure developed in the present study demonstrated that monodisperse nanocrystals of bimetallic oxide could be obtained directly without a sizeselection process.

Acknowledgment. We are grateful to the Creative Research Initiative Program and the Center for Advanced Materials Processing of the Korean Ministry of Science and Technology for financial support. We thank Prof. Kibum Kim and Mr. Jihoon Oh for their kind help in TEM studies.

Supporting Information Available: XRD pattern of $6 \mathrm{~nm}$ iron-cobalt alloy nanoparticles after heating under argon at 500 ${ }^{\circ} \mathrm{C}$; TEM image of a three-dimensional superlattice of $6 \mathrm{~nm}$ cobalt ferrite nanocrystals; HRTEM image of $9 \mathrm{~nm}$ cobalt ferrite nanocrystals; TEM images of 8 and $4 \mathrm{~nm}$ cobalt ferrite nanocrystals. This material is available free of charge via the Internet at http://pubs.acs.org.

\section{References and Notes}

(1) (a) Cornell, R. M.; Schwertmann, U. The iron oxides; VCH: Weinheim, Germany, 1996. (b) Fertman, V. E. Magnetic Fluids Guidebook: Properties and Applications; Hemisphere Publishing Co.: New York, 1990. (c) Berkovsky, B. M.; Medvedev, V. F.; Krakov, M. S. Magnetic Fluids: Engineering Applications; Oxford University Press: Oxford, U.K., 1993.

(2) (a) Awschalom, D. D.; DiVicenzo, D. P. Phys. Today 1995, 4, 43 (b) Leslie-Pelecky, D. L.; Rieke, R. D. Chem. Mater. 1996, 8, 1770. (c) Raj, K.; Moskowitz, R. J. Magn. Magn. Mater. 1990, 85, 233. (d) Speliotis, D. E. J. Magn. Magn. Mater. 1999, 193, 29.

(3) (a) Park, S.-J.; Kim, S.; Lee, S.; Khim, Z. G.; Char, K.; Hyeon, T. J. Am. Chem. Soc. 2000, 122, 8581. (b) Puntes, A. F.; Krishnan, K. M.; Alivisatos, A. P. Science 2001, 291, 2115. (c) Sun, S.; Murray, C. B. J. Appl. Phys. 1999, 85, 4325. (d) Sun, S.; Murray, C. B.; Weller, D.; Folks, L.; Moser, A. Science 2000, 287, 1989. (e) Suslick, K. S.; Fang, M.; Hyeon, T. J. Am. Chem. Soc. 1996, 118, 11960.

(4) (a) Fried, T.; Shemer, G.; Markovich, G. Adv. Mater. 2001, 13, 1158. (b) Moumen, N.; Pileni, M. P. Chem. Mater. 1996, 8, 1128. (c) Ngo, A. T.; Pileni, M. P. Adv. Mater. 2000, 12, 276. (d) Liu, C.; Zou, B.; Rondinone, A. J.; Zhang, Z. J. J. Am. Chem. Soc. 2000, 122, 6263. (e) Tang, Z. X.; Sorensen, C. M.; Klabunde, K. J.; Hadjipanayis, G. C. J. Colloid Interface Sci. 1991, 146, 38. (f) Chen, J. P.; Lee, K. M.; Sorensen, C. M.; Klabunde, K. J.; Hadjipanayis, G. C. J. Appl. Phys. 1994, 75, 5876.

(5) (a) Rockenberger, J.; Scher, E. C.; Alivisatos, A. P. J. Am. Chem. Soc. 1999, 121, 11595. (b) Tamura, H.; Matijevic, E. J. Colloid Interface Sci. 1982, 90, 100. (c) Ziolo, R. F.; Giannelis, E. P.; Weinstein, B. A.; O’Horo, M. P.; Ganguly, B. N.; Mehrotra, V.; Russell, M. W.; Huffman, D. R. Science 1992, 257, 219. (d) Shafi, K. V. P. M.; Gedanken, A. Chem Mater. 1998, 10, 3445 .

(6) Hyeon, T.; Lee, S. S.; Park, J.; Chung, Y.; Na, H. B. J. Am. Chem. Soc. 2001, 123, 12798.

(7) Knight, J.; Mays, M. J. J. Chem. Soc. A 1970, 654.

(8) Rondinone, A. J.; Samia, A. C. S.; Zhang, Z. J. J. Phys. Chem. B 1999, $103,6876$.

(9) Venugopal, R.; Sundaravel, B.; Cheung, W. Y.; Wilson, I. H.; Wang, F. W.; Zhang, X. X. Phys. Rev. B 2001, 65, 14418.

(10) Leslie-Pelecky, D. L.; Rieke, R. D. Chem. Mater. 1996, 8, 1770.

(11) Buschow, K. H. J., Ed. Handbook of Magnetic Materials; NorthHolland: Amsterdam, 1995; Vol. 8, p 212

(12) Rondinone, A. J.; Samia, A. C. S.; Zhang, Z. J. Appl. Phys. Lett. 2000, 76, 3624 